\title{
Descriptions of Memory Rehabilitation Group Interventions for Neurological conditions: A systematic review
}

\begin{tabular}{|c|c|}
\hline Journal: & Clinical Rehabilitation \\
\hline Manuscript ID: & CRE-2015-4288.R1 \\
\hline Manuscript Type: & Original Article \\
\hline Keywords: & Cognitive Rehabilitation, Memory Rehabilitation, systematic review \\
\hline Abstract: & $\begin{array}{l}\text { Objective: To establish what aspects of group-based cognitive } \\
\text { rehabilitation for memory problems are reported, and to develop a } \\
\text { checklist for authors, which may to improve reporting of these } \\
\text { interventions in future studies. } \\
\text { Data sources: A systematic search was conducted on Web of Knowledge, } \\
\text { CINAHL, MEDLINE, AMED, EMBASE and PsycINFO electronic databases } \\
\text { (last search: 01/05/2015). } \\
\text { Review methods: Articles were included if the sample were adults with a } \\
\text { neurological disorder, the intervention was group-based cognitive } \\
\text { rehabilitation for memory problems, and if the study was a randomised } \\
\text { controlled trial. Articles were independently screened for inclusion and data } \\
\text { extracted by two researchers, with the third researcher arbitrating any } \\
\text { disputes. } \\
\text { Results: Fourteen studies were included in this review. The reporting of } \\
\text { certain aspects of an intervention was found to be poor, particularly in } \\
\text { relation to: duration of the programme ( } 6 \text { of } 14 \text { studies did not report), the } \\
\text { development of the intervention ( } 7 \text { of } 14 \text { studies did not discuss), and the } \\
\text { content and structure of intervention ( } 7 \text { of the } 14 \text { studies did not provide } \\
\text { details). } \\
\text { Conclusion: This review found that the overall reporting of memory } \\
\text { rehabilitation content and format is poor. Refinement and adaption of pre- } \\
\text { existing checklists to capture aspects of cognitive rehabilitation } \\
\text { programmes may help authors when reporting complex interventions. A } \\
\text { draft checklist is provided that could be refined and validated in further } \\
\text { research. }\end{array}$ \\
\hline
\end{tabular}

\section{SCHOLARONE ${ }^{m}$ \\ Manuscripts}




\section{Abstract}

Objective: To establish what aspects of group-based cognitive rehabilitation for memory problems are reported, and to develop a checklist for authors, which may to improve reporting of these interventions in future studies.

Data sources: A systematic search was conducted on Web of Knowledge, CINAHL, MEDLINE, AMED, EMBASE and PsycINFO electronic databases (last search: 01/05/2015).

Review methods: Articles were included if the sample were adults with a neurological disorder, the intervention was group-based cognitive rehabilitation for memory problems, and if the study was a randomised controlled trial. Articles were independently screened for inclusion and data extracted by two researchers, with the third researcher arbitrating any disputes.

Results: Fourteen studies were included in this review. The reporting of certain aspects of an intervention was found to be poor, particularly in relation to: duration of the programme ( 6 of 14 studies did not report), the development of the intervention (7 of 14 studies did not discuss), and the content and structure of intervention ( 7 of the 14 studies did not provide details).

Conclusion: This review found that the overall reporting of memory rehabilitation content and format is poor. Refinement and adaption of pre-existing checklists to capture aspects of cognitive rehabilitation programmes may help authors when reporting complex interventions. A draft checklist is provided that could be refined and validated in further research. 
Introduction

Establishing the effectiveness of group-based memory rehabilitation programmes has been the aim of several recent randomised controlled trials (RCTs). While there is good evidence of effectiveness in some conditions, such as traumatic brain injury (TBI; e.g. (1)), in other conditions the evidence is still equivocal (e.g., in MS (2); stroke (3)). The differences in effectiveness found in the various studies reflect the heterogeneity in patient groups (including severity of condition), the outcomes measured, the methodological and statistical quality of the study, and of course, the intervention itself. While aspects such as patient groups included, or outcomes measured can be determined form most manuscripts, perhaps due to the enforcement of the CONSORT statement (4) by some journals, the intervention itself is not always explicitly described. In fact, a systematic review by van Heugten et al. (5) arrived at this very conclusion in their summary of 95 RCTs of cognitive rehabilitation.

Presenting sufficient details of the intervention undertaken in a report of an RCT is important to appraise, replicate, or improve the study. Firstly, astute readers who have clinical or research expertise in the area may be able to appraise the intervention and the resultant findings of the RCT. Secondly, if an intervention has been found to be effective, clinicians will need to have sufficient information to ensure they are able to replicate the study, and by extension, the results to ensure that such interventions are commissioned in healthcare systems. Furthermore, replicability is also important from a research perspective to ensure that the findings of the trial are not due to error or bias. Finally, for trials that find effectiveness and for those that do not, there may be ways to improve the intervention. For positive trials, for instance, improvements could focus on reducing participant burden in terms of time, or cost-savings in terms of alternative formats of delivering the intervention. For negative trials, researchers can appraise the intervention and modify it so as to not try the same intervention on another group of participants, which could be a waste of resources in terms of finance, time, and effort.

The recently published Template for Intervention Description and Replication (TIDieR) checklist (6) was developed on the basis of a literature review that found that the quality of descriptions of interventions 'remarkably poor', and used the Delphi process to produce a 12-item general checklist for reporting of interventions. Whilst the TIDieR guidelines may improve the reporting of interventions, this checklist is not 
specific to group-based psychological or rehabilitation interventions, but a general guide for studies of all interventions, including pharmacological studies. While the generality of the guide can be a strength, we believe that cognitive rehabilitation-specific guidelines for group-based interventions may further encourage authors to ensure sufficient details of the intervention are reported.

Our review builds on the aforementioned van Heugten et al. (5) systematic review in several ways. Firstly, we have updated the search (their review only covered studies published up to 2010) and have focussed specifically on one cognitive domain, i.e. memory. We are in complete agreement with van Heugten et al. (5) and Hoffman et al. (6) in their assessment that there is an urgent need to have a checklist agreed upon by both clinicians and researchers in the field of cognitive rehabilitation. However, given the heterogeneity of cognitive rehabilitation domains and intervention types and formats, we may need to go beyond characteristics which are considered 'basic minimum' in reporting of trials, to more nuanced cognitive domain-specific recommendations; hence our focus on memory interventions here. Secondly, we wanted to assess whether there were additional categories that we could add to a checklist for assessing group-based memory rehabilitation.

Method

A systematic search was conducted on the following electronic bibliographic databases from their inception, and the last date searched was 01/05/2015: Web of Knowledge, Cumulative Index to Nursing and Allied Health (CINAHL), MEDLINE, Allied and Complementary Medicine Database (AMED), EMBASE and PsycINFO. A search strategy was created for MEDLINE and amended for each database (see Appendix A for details).

Our search strategy was developed by two researchers (KM \& ES) in consultation with the third researcher $(\mathrm{RdN})$, and was based on the following inclusion/exclusion criteria: We included studies that had adult patients with any neurological condition (of single or multiple diagnoses), who received memory rehabilitation (or cognitive rehabilitation with a specific focus on memory), and delivered in a group format (defined as more than two participants). The study had to be an RCT. The following hierarchy was used when considering which studies to exclude: Not RCT, not a neurological disorder, not focussed on memory, non-intervention papers, not a group intervention, intervention not patient-focussed (i.e., it was for carers or staff), not adults. Studies were also excluded if they were a re-analysis or sub-group analysis of primary studies, or were a sub-study of 
an included trial. As the aim of this review was to examine the way memory interventions are described within a paper, the quality of study itself was not taken into account.

Papers were screened independently by two researchers (KM \& ES) using the title and abstract. In cases where the abstract provided insufficient detail, full papers were accessed. If there was disagreement regarding the inclusion or exclusion of a paper, a third researcher (RdN) arbitrated. See figure 1 for the PRISMA (Preferred Reporting Items for Systematic Reviews and Meta-Analyses(7)) flow diagram.

Figure 1 here

The description of the intervention of each paper was extracted and this was coded independently by KM and ES. Codes were developed in two ways: (i) by reviewing the previous systematic review by van Heugten et al. (5), examining their data extraction technique and the consensus they gained from their review, and (ii) by considering what information, as clinicians and researchers working in this area, we would have needed to replicate an intervention in clinical practice or research. The codes were then discussed between all authors and a final list of codes was agreed on. Any disagreements were arbitrated by RdN. Each reviewer assigned a code to each segment of text which they believe adequately and succinctly described that section of text. The rest of each individual paper was then checked against these final codes to ensure that the code was appropriate to the relevant text and that key information (e.g., diagnosis of group) was not presented elsewhere within the paper.

The checklist was developed on the basis of our results, which combined the codes we had attributed for key aspects describing the intervention in individual studies.

\section{Results}


1

2

3

4

5

6

7

8

9

10

11

12

13

14

15

16

17

18

19

20

21

22

23

24

25

26

27

28

29

30

31

32

33

34

35

36

37

38

39

40

41

42

43

44

45

46

47

48

49

50

51

52

53

54

55

56

57

58

59

60
The characteristics of the included studies are presented in table 1.

Table 1 here

Our review included 14 studies, totalling 990 participants.

The descriptions of the interventions in each study were coded and these used to create a table highlighting the main 'ingredients' of the intervention for each study. This is presented in table 2.

Table 2 here

As can be seen from table 2, overall there were six main codes, which emerged from the review. Five of these were divided into sub-codes, depicting the key contents of the main codes.

Groups

Seven of the 14 studies (8-14) did not report any information relating to the size, or size range, of the groups.

Of the seven studies that did provide this information (15-21), group sizes ranged from 3 (18) to 15 (19).

However, all of the studies included information of their participants' specific diagnosis that was related to their memory problems.

\section{Aim/Focus}


Ten of the studies did not give the specific aim of the group sessions $(2,9,10,12,15-20)$, but only reported the general aim of improving memory and associated cognitive domains. Four studies $(8,11,14,21)$ explicitly reported the overall aim or focus of the group sessions. These were to: build on efficacy for use of compensatory strategies (11), subjective memory experiences (8) alongside themes using reminiscence (14), and to examine multiple aspects of cognition to impact global memory and executive function (21).

Dose

Three of the 14 included studies $(9,20,21)$ did not report the overall number of sessions within the group programmes, and one of these (9) did not report the length of each session. Of the 11 studies that included number of sessions within a programme; 6 was the fewest $(10,19)$ and 14 the largest number of sessions $(14$, 16). Four of the 14 studies $(9,12,13,15)$ did not give any indication of the frequency of the group sessions; of those which did include this detail, session frequency was twice weekly for six studies $(8,14,16-18,21)$, weekly in three studies $(10,11,19)$ and bi-weekly in one $(20)$. Six studies $(8,10,11,13,17,21)$ did not report the overall duration of the entire group programme, but in studies where this was reported, programme durations ranged from 6 weeks $(9,19)$ to 6 months $(12,20)$.

Programme

Only one study (21) reported that their group intervention was an original programme that the authors had developed, whereas five studies $(8,14-16,19)$ reported that the group intervention within their trial was adapted from previous research. Of these five studies, four of the trials $(8,14,16,19)$ provided a reference to the original programme, from which the intervention was developed or adapted. One study (18) had two intervention groups that were compared to a control, where one of the experimental interventions was a novel programme designed for the trial whilst the other was a previously developed programme, for which a reference was provided.

Four of the studies $(13,15,18,19)$ reported that theirs was a manual-based programme, but only two of these manuals $(15,18)$ were accessible. Three of the four studies using a manual $(13,15,18)$ reported how the manual was developed. 
Although a manual was not used, or not readily accessible, three of the studies $(11,12,19)$ gave session-bysession content, i.e., these three studies gave a clear indication of the topics covered in each group session. Only one study (15) reported how missed sessions were dealt with.

\section{Format of Sessions}

Seven of the included studies $(9,11-13,18,19,21)$ reported details on the general format of the sessions, e.g., start with a recap of the previous session, end with a summary, etc., with three studies providing this information in a table $(9,12,19)$. Three of the studies $(8,10,19)$ reported that the members of the intervention groups were given materials/booklets to work through during the programme. Three studies (9, $13,19)$ reported that participants were given a break during each session, and eight trials $(8-13,15,19)$ reported that participants were set homework as part of the intervention.

\section{Facilitator}

Three of the included studies $(14,17,18)$ did not report any details about who facilitated the group sessions, e.g., whether it was ran by an Occupational Therapist, Research Assistant, Clinical Neuropsychologist, etc., and whether any specific form of training was provided to the facilitators. Of those that included information about who facilitated the programme, the most common was 'Psychologists' $(8,9,12)$, with two studies using Research Assistants $(13,21)$. Others had a "Masters prepared Nurse"(11), Neuropsychologist (19), Clinical Geropsychologist (10), Assistant Psychologist trained by a Clinical Psychologist (15), Activity Co-ordinator (16) and Neurophysiologists, with student assistants (20), as facilitators.

\section{Discussion}

Our aim was to establish what aspects of group-based cognitive rehabilitation for memory problems are reported in clinical trials, with a view to develop a checklist for authors. The overall reporting of interventions was poor. Providing clear descriptions of interventions is vital for researchers and clinicians to: (i) make informed decisions about whether a particular intervention would be applicable to their client group and their service/clinic, and (ii) determine how the evaluated intervention can be adapted for clinical use, without compromising the integrity of the intervention - as it was tested. However, as can be seen from our results, 
often relatively few details were provided. For instance, four of the included studies reported no details about who facilitated the group programme. This problem has been found to exist in other domains of cognitive rehabilitation research also, with van Heugten et al. (5) in their systematic review of content of cognitive rehabilitation interventions for acquired brain injuries finding no papers that reported the expertise or competences of the treating staff. Without this information it would be difficult to assess what kind of staffing and training resources are required to successfully run such interventions.

Although four of the studies were manual-based, only two of these manuals were readily accessible. Authors could make explicit how to access the manual (even if this was not for free), should the reader wish to find out more information. If the intervention was not manualised, authors should attempt to provide an outline of the intervention, identifying its key elements. This was done in three studies $(11,12,19)$ using a table to present this information. Another good example was a three-armed trial (17), comparing two interventions (one novel, one manualised) against a control. This study gave references for the manual as well as supplying supplementary information that outlined both the manualised and the novel intervention, making it easy for the reader to gain a clear understanding of what was being tested.

Another noteworthy aspect of our findings was that very few studies reported basic information about how the intervention can be delivered in or adapted for regular clinical practice, which would demand a certain degree of flexibility without changing the intervention. For example, only one study (15) provided details on how missed sessions were dealt with. While it is common practice to describe how missing data was dealt with during a statistical analysis, we believe that when reporting group-based programmes, issues such as how missed sessions were addressed, or delays in the weekly sessions could be reported in similarly rigorous ways.

One possible reason for the lack of detail provided in most papers is the limited word-count allowed by some journals, particularly medical journals. However, the advent of online publications that offer the option of uploading supplementary material provides additional possibilities of including this information, which was done by one study (18). Furthermore, some authors have begun to explore graphical adjuncts to textual descriptions of content of information, thereby allowing greater flexibility to present this information (see(22)). 
The purpose of this review was not to comment on the quality of any of the studies included, nor was it to comment on what should be included as part of a successful group-based intervention. The purpose of this review was to identify what is currently reported in trials of complex interventions evaluating memory rehabilitation, and use this as a basis for creating a checklist for authors to use as an aide memoire when reporting complex intervention trials. Using the codes and sub-codes from the data extracted as a starting point, we created a checklist of aspects of an intervention that was commonly reported, or at least referred to, in the write-up of a complex intervention (see Appendix B). This checklist should be seen as an adjunct to the one presented in van Heugten et al. ((5), p666), and a more cognitive rehabilitation-specific adaption of the TIDieR checklist (6). While the Van Heugten et al. (5) paper is more comprehensive in scope (suggesting for example what patient characteristics and treatment goals, in addition to the intervention characteristics to include), ours is more focussed on group interventions. Therefore, while they ask the authors to specify whether the intervention was provided in group or individual format, ours requests authors to report what the group size and composition were.

As there were only 14 studies included in this review, all of which were RCTs, it is possible that there are components of a memory rehabilitation programme which are not included in the checklist. The checklist proposed will need to be evaluated against studies using other research designs describing these complex interventions to see whether there are any additional items that need to be added to the checklist. It is also possible that some of the items on the checklist may not be absolutely necessary for researchers and clinicians to make informed decisions about a particular intervention; however, as the checklist is in its infancy, we felt it was necessary to be over-inclusive rather than missing potentially important aspects.

The findings of this study need to be considered in light of the limitations of this review. Like most systematic reviews, despite our extensive search strategy we may have missed some trials. However, we mitigated this by cross-checking reference lists of all identified papers and systematic reviews of cognitive rehabilitation. Our focus was limited to RCTs of memory rehabilitation programmes that were delivered in group formats; therefore, our findings only represent one aspect of cognitive rehabilitation, and rehabilitation studies in other domains, such a visual neglect or executive functions, may be more explicit in their description of content of intervention. However, the findings from the van Heugten et al. (5) review would suggest otherwise. 


\section{Future directions}

We regard this review as another step in the movement towards developing more refined checklists in the area of cognitive rehabilitation. We agree with van Heugten et al. (5) and Hoffman et al. (6) that to have a standard checklist that could be adapted for specific cognitive domains is a good way to improve reporting of cognitive rehabilitation. One possible way to further develop this checklist would be to follow the protocol used to produce the TIDieR guidelines (6) and use a Delphi consensus method with professionals who deliver memory rehabilitation to find out from them what they feel would be the most significant aspects of an intervention that would need to be described (and in what level of detail) to enable them to deliver it in clinical settings.

\section{Clinical Messages}

- Currently, group-based memory rehabilitation interventions are poorly described.

- Trialists should ensure that session by session content, manuals, dose and general session formats are described in order to allow researchers to duplicate the studies, and clinicians to implement them as they were delivered in the trial.

- The use of intervention description checklists may improve reporting of complex intervention in future studies.

\section{Author contributions}

KJM developed the search strategy, ran the search and identified studies for inclusion, completed data analysis and contributed to the write up of the article.

ES helped with the search strategy, identified studies for inclusion, and completed data analysis.

RdN oversaw the development of the search strategy, identification of studies to be included and data analysis, and contributed to the write of the article 


\section{Competing interests}

RdN is an author of two studies that were included in this review. 


\section{References}

1. Cicerone KD, Langenbahn DM, Braden C, Malec JF, Kalmar K, Fraas M, et al. Evidence-based cognitive rehabilitation: updated review of the literature from 2003 through 2008. Arch Phys Med Rehabil. 2011 Apr;92(4):519-30. PubMed PMID: 21440699.

2. Das Nair R, Ferguson H, Stark D, Lincoln N. Memory Rehabilitation for People with Multiple Sclerosis. Cochrane Database of Systematic Reviews. 2012 (3).

3. Das Nair R, lincoln N. Cognitive Rehabilitation for Memory Deficits following Stroke. Cochrane Database of Systematic Reviews. 2008 (4).

4. Moher D, Schulz K, Altman D. The CONSORT statement: revised recommendations for improving the quality of reports of aprallel group randomized trials. BMC Medical Research Methodology. 2001;1(2).

5. van Heugten C, Gregorio GW, Wade D. Evidence-based cognitive rehabilitation after acquired brain injury: a systematic review of content of treatment. Neuropsychol Rehabil. 2012;22(5):653-73. PubMed PMID: 22537117.

6. Hoffmann TC, Glasziou PP, Boutron I, Milne R, Perera R, Moher D, et al. Better reporting of interventions: template for intervention description and replication (TIDieR) checklist and guide. BMJ. 2014;348:g1687. PubMed PMID: 24609605.

7. Moher D, Liberati A, Tetzlaff J, Altman DG. Preferred reporting items for systematic reviews and metaanalyses: the PRISMA statement2009 2009-07-21 10:46:49.

8. Aben L, Heijenbrok-Kal MH, van Loon EM, Groet E, Ponds RW, Busschbach JJ, et al. Training memory self-efficacy in the chronic stage after stroke: a randomized controlled trial. Neurorehabil Neural Repair. 2013 Feb;27(2):110-7. PubMed PMID: 22895620.

9. Jennett S, Lincoln N. An evaluation of the effectiveness of group therapy for memory problems. Int Disabil Stud. 1991 Jul-Sep;13(3):83-6. PubMed PMID: 9108633. 
10. Rapp S, Brenes G, Marsh AP. Memory enhancement training for older adults with mild cognitive impairment: a preliminary study. Aging Ment Health. 2002 Feb;6(1):5-11. PubMed PMID: 11827617.

11. Stuifbergen AK, Becker H, Perez F, Morison J, Kullberg V, Todd A. A randomized controlled trial of a cognitive rehabilitation intervention for persons with multiple sclerosis. Clin Rehabil. 2012 Oct;26(10):882-93. PubMed PMID: 22301679.

12. Troyer AK, Murphy KJ, Anderson ND, Moscovitch M, Craik FI. Changing everyday memory behaviour in amnestic mild cognitive impairment: a randomised controlled trial. Neuropsychol Rehabil. 2008 Jan;18(1):65-

88. PubMed PMID: 17943615.

13. das Nair R, Lincoln NB. Evaluation of rehabilitation of memory in neurological disabilities (ReMiND): a randomized controlled trial. Clin Rehabil. 2012 Oct;26(10):894-903. PubMed PMID: 22324056.

14. Spector A, Thorgrimsen L, Woods B, Royan L, Davies S, Butterworth M, et al. Efficacy of an evidencebased cognitive stimulation therapy programme for people with dementia: randomised controlled trial. The British journal of psychiatry : the journal of mental science. 2003 Sep;183(3):248-54. PubMed PMID: 12948999.

15. Carr SE, das Nair R, Schwartz AF, Lincoln NB. Group memory rehabilitation for people with multiple sclerosis: a feasibility randomized controlled trial. Clin Rehabil. 2014 Jan 22. PubMed PMID: 24452705. Epub 2014/01/24. Eng.

16. Coen RF, Flynn B, Rigney E, O'Connor E, Fitzgerald L, Murray C, et al. Efficacy of cognitive stimulation therapy programme for people with dementia. Irish Journal of Psychological Medicine. 2011 Sep;28(3):145-7.

17. Olchik MR, Farina J, Steibel N, Teixeira AR, Yassuda MS. Memory training (MT) in mild cognitive impairment (MCl) generates change in cognitive performance. Arch Gerontol Geriatr. 2013 May-Jun;56(3):4427. PubMed PMID: 23260332.

18. Petrelli A, Kaesberg S, Barbe MT, Timmermann L, Fink GR, Kessler J, et al. Effects of cognitive training in Parkinson's disease: A randomized controlled trial. Parkinsonism \& Related Disorders. 2014 Nov;20(11):1196-202. PubMed PMID: 2014-45326-004. English. 
19. Radford K, Lah S, Thayer Z, Say MJ, Miller LA. Improving memory in outpatients with neurological disorders using a group-based training program. Journal of the International Neuropsychological Society : JINS. 2012 Jul;18(4):738-48. PubMed PMID: 22594962.

20. Rojas GJ, Villar V, Iturry M, Harris P, Serrano CM, Herrera JA, et al. Efficacy of a cognitive intervention program in patients with mild cognitive impairment. International psychogeriatrics / IPA. 2013 May;25(5):82531. PubMed PMID: 23414646.

21. Eckroth-Bucher $M$, Siberski J. Preserving cognition through an integrated cognitive stimulation and training program. Am J Alzheimers Dis Other Demen. 2009 Jun-Jul;24(3):234-45. PubMed PMID: 19346501.

22. Perera P, Heneghan C, Yudkin P. A graphical method for depicting randomised trials of complex interventions. BMJ. 2007;33:127-9 
Table 1: Details of the studies included

\begin{tabular}{|c|c|c|c|}
\hline Reference No. & Author(s) & Country & Sample: Diagnosis, sample size \\
\hline 8 & Aben et al., 2013 & The Netherlands & Stroke, $n=153$ \\
\hline 9 & Jennet \& Lincoln 1991 & UK & Stroke, $n=8 ; C H I, n=10$ \\
\hline 10 & Rapp, Brenes \& Marsh 2002 & USA & $\mathrm{MCl}, \mathrm{n}=19$ \\
\hline 11 & Stuifbergen 2012 & USA & $M S, n=63$ \\
\hline 12 & Troyer et al. 2008 & Canada & $\mathrm{aMCl}, \mathrm{n}=68$ \\
\hline 13 & dasNair \& Lincoln 2012 & UK & MS, n=39; Stroke, n=17; TBI, n= 16 \\
\hline 14 & Spector, et al. 2003 & UK & Dementia, $\mathrm{n}=201$ \\
\hline 15 & Carr et al., 2014 & UK & MS, $n=48$ \\
\hline 16 & Coen et al., 2011 & Ireland & Dementia, $n=27$ \\
\hline 17 & Olchik et al. 2012 & Brazil & Healthy controls, $n=65 ; \mathrm{MCl}, \mathrm{n}=47$ \\
\hline 18 & Petrelli et al. 2014 & Germany & Parkinson's Disease, $\mathrm{n}=90$ \\
\hline 19 & Radford et al. 2012 & Australia & $\begin{array}{l}\text { Epilepsy, } n=19 ; \text { Stroke, } n=21 ; \text { Tumour/Cyst, } n=10 ; T B I, n=2 \text {; } \\
\text { Encephalitis, } n=2 ; \text { Hydrocephalus, } n=1, S L E, n=1\end{array}$ \\
\hline 20 & Rojas et al. 2013 & Argentina & $\mathrm{MCl}, \mathrm{n}=46$ \\
\hline 21 & Ecroth-Bucher \& Siberski 2009 & USA & Alzheimer's, $n=37$ \\
\hline
\end{tabular}

Note: MS (multiple sclerosis), TBI (traumatic brain injury), $\mathrm{CHI}$ (closed head injury), $\mathrm{MCl}$ (mild cognitive impairment), aMCl (amnestic mild cognitive impairment), SLE (systemic lupus erythematosus) 
Table 2: Description of studies that endorse various codes.

\begin{tabular}{|c|c|c|c|c|c|c|c|c|c|c|c|c|c|c|c|}
\hline \multirow[t]{2}{*}{ Code } & \multirow[t]{2}{*}{ Sub-code } & \multicolumn{14}{|c|}{ Reference number for study } \\
\hline & & 8 & 9 & 10 & 11 & 12 & 13 & 14 & 15 & 16 & 17 & 18 & 19 & 20 & 21 \\
\hline \multirow[t]{4}{*}{ Dose } & Number of sessions & + & & + & + & + & + & + & + & + & + & + & + & & \\
\hline & Length of session & + & & + & + & + & + & + & + & + & + & + & + & + & + \\
\hline & Frequency of sessions & + & & + & + & & & + & & + & + & + & + & + & + \\
\hline & Duration of programme & & + & & & + & & + & + & + & & + & + & + & \\
\hline \multirow[t]{2}{*}{ Group } & Size & & & & & & & & + & + & + & + & + & + & + \\
\hline & Diagnosis & + & + & + & + & + & + & + & + & + & + & + & + & + & + \\
\hline Aim/Focus & & + & & & + & & & + & & & & & & & + \\
\hline \multirow[t]{8}{*}{ Programme } & Original? & 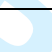 & & & & & & & & & & + & & & + \\
\hline & Adapted? & + & & & & & & + & + & + & & + & + & & \\
\hline & Manualised? & & $\overline{2}$ & & & & + & & + & & & + & + & & \\
\hline & Manual available? & & 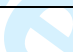 & 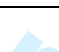 & & & & & + & & & + & & & \\
\hline & $\begin{array}{l}\text { How was manual } \\
\text { developed/adapted? }\end{array}$ & & $v^{2}$ & & & & + & & + & & & + & & & \\
\hline & $\begin{array}{l}\text { If adapted, are clear refs given to } \\
\text { original programme? }\end{array}$ & + & & & & & & + & & + & & + & + & & \\
\hline & $\begin{array}{l}\text { Session by Session content } \\
\text { reported }\end{array}$ & & & & + & + & H & 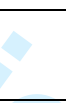 & & & & + & + & & \\
\hline & $\begin{array}{l}\text { How are missed sessions dealt } \\
\text { with? }\end{array}$ & & & & & & 4 & & + & & & & & & \\
\hline Facilitator & Who facilitated? & + & + & + & + & + & + & & + & + & & & + & + & + \\
\hline \multirow[t]{4}{*}{ Format of sessions } & $\begin{array}{l}\text { General format of session reported } \\
\text { (overview, summary, etc.) }\end{array}$ & & + & & + & + & + & & 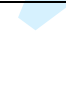 & & & + & + & & + \\
\hline & Booklet/materials given? & + & & + & & & & & & & & & + & & \\
\hline & Break & & + & & & & + & & & & & & + & & \\
\hline & Was homework set? & + & + & + & + & + & + & & + & & & & + & & \\
\hline
\end{tabular}

8. Aben et al. 2013; 9. Jennet \& Lincoln 1991; 10. Rapp, Brenes \& Marsh 2002; 11. Stuifbergen 2012; 12. Troyer et al. 2008; 13. das Nair \& Lincoln 2012; 14. Spector et al. 2003; 15. Carr et al. 2014; 16. Coen et al. 2011; 17. Olchik et al. 2012; 18. Petrelli et al. 2014; 19. Radford et al. 2012; 20. Rojas et al. 2013; 21. Ecrocth-Bucher \& Siberski 2009. 
1

2

3

4

5

6

7

8

9

10

11

12

13

14

15

16

17

18

19

20

21

22

23

24

25

26

27

28

29

30

31

32

33

34

35

36

37

38

39

40

41

42

43

44

45

46

47

48

49

50

51

52

53

54

55

56

57

58

59

60

Figure 1: PRISMA Diagram

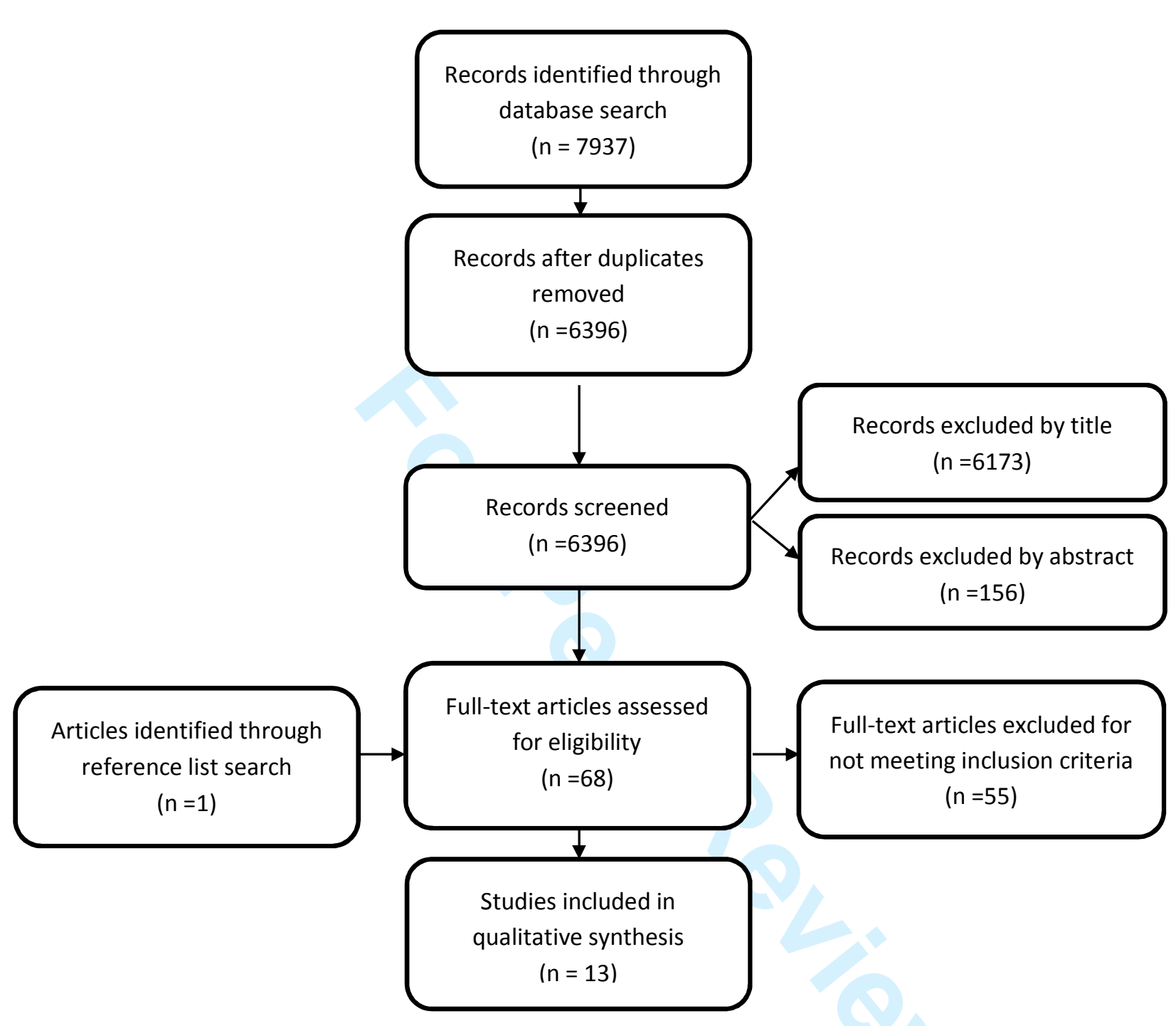


Appendix A

1. exp Brain Damage/ or exp Traumatic Brain Injury/ or exp Head Injuries/

2. exp stroke/ or brain infarcation/

3. exp Multiple Sclerosis/ or demyelinating diseases/

4. exp Presenile Dementia/ or exp Dementia/ or exp Senile Dementia/ or exp AIDS Dementia Complex/ or exp Semantic Dementia/ or exp Dementia with Lewy Bodies/ or exp Vascular Dementia/

5. exp Epilepsy/

6. exp Hematoma/

7. exp Aneurysms/

8. (craniocerebral trauma\$ or acquired brain injur* or traumatic brain injur* or stroke or anoxia or hypoxi\$ or multiple sclerosis or ms or demyelinating autoimmune disease $\$$ or dementia or alzheimers or epilepsy or tumour\$ or h?ematoma or aneurysm or h?emorrhag* or encephaliti* or mening* or emboli* or amnesia or korsakoffs or neurological).af.

9. exp cognition/ or exp memory/ or exp cognition disorders/ or exp memory disorders/

10. (cognitive or cognition or memory).af.

11. (training or re-training or retraining or therap\$ or rehabilitation or treatment\$ or therapeutic\$ or computer-assisted therap\$ or computer assisted therap\$).af.

12. exp rehabilitation/

13. exp Cognitive Therapy/

14. exp computers/

15. exp Computer Assisted Therapy/

16. exp Neuropsychological Tests/

17. (neurorehabilitation or neuropsychological rehabilitation or cognitive rehabilitation or memory rehabilitation or cognitive retraining).af.

18. exp Randomized Controlled Trials/

19. randomized controlled trials.mp. [ $\mathrm{mp}=$ title, abstract, original title, name of substance word, subject heading word, keyword heading word, protocol supplementary concept word, rare disease supplementary concept word, unique identifier]

20. (random allocation or placebo\$).mp.

21. controlled clinical trials.mp. 
22. (random\$ or placebo\$).tw.

23. (controlled adj5 (trial\$ or stud\$)).tw.

24. (clinical\$ adj5 trial\$).tw.

25. 1 or 2 or 3 or 4 or 5 or 6 or 7 or 8

26. 9 or 10

27. 11 or 12 or 13 or 14 or 15 or 16 or 17

28.18 or 19 or 20 or 21 or 22 or 23 or 24

29. Group.af

30. 25 and 26 and 27 and 28 and 29

31. limit 30 to (humans and ("young adult (19 to 24 years)" or "adult (19 to 44 years)" or "young adult and adult (19-24 and 19-44)" or "middle age (45 to 64 years)" or "middle aged (45 plus years)" or "all aged (65 and over)" or "aged (80 and over)")) 
Appendix B

Checklist for writing descriptions of complex interventions.

Have you...

Described the dose of intervention?
Number of sessions
Duration of session
Frequency of sessions
Duration of the whole programme
Described group characteristics?
Group size
Mixed or single diagnosis groups

Described the aim/focus of the intervention?

Described the intervention programme itself?

Was the intervention original?

$\square$ Was the intervention adapted?

$\square$ Are details given on how the programme was developed/adapted?

$\square$ Is session by session content described?

$\square$ Is the intervention manualised?

$\square$ Is the intervention manual available?

$\square$ How are missed sessions dealt with?

Described who facilitated the groups?

How many facilitators where there?

What kind of training did they receive?

Described the format of the sessions?

General format of session (overview, summary, preview, etc.)

Was a booklet/other materials given to participants?

Was there a break?

Was homework set?

If homework was set, has this been described?

$\square$ How/when was this evaluated/discussed in the group? 


\section{Abstract}

Objective: To establish what aspects of group-based cognitive rehabilitation for memory problems are reported, and to develop a checklist for authors, which may to improve reporting of these interventions in future studies.

Data sources: A systematic search was conducted on Web of Knowledge, CINAHL, MEDLINE, AMED, EMBASE and PsycINFO electronic databases (last search: 01/05/2015).

Review methods: Articles were included if the sample were adults with a neurological disorder, the intervention was group-based cognitive rehabilitation for memory problems, and if the study was a randomised controlled trial. Articles were independently screened for inclusion and data extracted by two researchers, with the third researcher arbitrating any disputes.

Results: Fourteen studies were included in this review. The reporting of certain aspects of an intervention was found to be poor, particularly in relation to: duration of the programme ( 6 of 14 studies did not report), the development of the intervention (7 of 14 studies did not discuss), and the content and structure of intervention ( 7 of the 14 studies did not provide details).

Conclusion: This review found that the overall reporting of memory rehabilitation content and format is poor. Refinement and adaption of pre-existing checklists to capture aspects of cognitive rehabilitation programmes may help authors when reporting complex interventions. A draft checklist is provided that could be refined and validated in further research. 